\title{
Sprachvermittlung und Spracherwerb in Afrika. Deutsch nach Englisch im südafrikanischen Kontext: Theorie und Praxis
}

\author{
Anne Baker \\ Department of German, Faculty of Humanities, University of Johannesburg, P.O. Box 524, Auckland Park 2006 \\ E-mail: abaker@uj.ac.za
}

\begin{abstract}
Teaching foreign languages in a multilingual context, such as one finds in South African schools, poses unique questions. The theory that pedagogically underpins and articulates the value of the study of foreign languages is internationally established and thus also valid in the South African context. Nevertheless, one has to consider language constellations in given multilingual circumstances in planning for the introduction and development of foreign languages in education, particularly where the primary language of learners is not a language of instruction and learning. This paper addresses the question of the desirability of teaching German as a foreign language to learners whose language of learning is not their first language and who have not yet mastered this language on an enabling level.
\end{abstract}

Keywords: multilingualism, language-constellation, metalinguistic and linguistic awareness, socioeconomic relationships

Schlüsselbegriffe: Multilingualismus, Sprachenkonstellation, metalinguistisches und linguistisches Bewusstsein, sozio-ökonomische Verhältnisse

\section{Einleitung}

Fremdsprachenvermittlung in einem multilingualen Kontext beruht auf mehreren theoretischen Grundlagen. An erster Stelle steht hier die Berücksichtigung der ,Sprachenkonstellation' der Lernenden (Missler 1999:24). Dabei sind die folgenden Aspekte von Bedeutung: Wie viele und welche Sprachen beherrschen die Lernenden und die Lehrenden, welche Kompetenz haben sie, welche gesellschaftliche Stellung kommt der L1 und der Zielsprache zu, wie steht es um die Sprachverwandtschaft der Ausgangssprachen mit der Zielsprache ${ }^{1}$ und welches metalinguistisches und linguistisches Bewusstsein (De Angelis 2007) haben die Lernenden und Lehrenden - ein komplexes Beziehungsgeflecht.

Außerdem sollen hier die Verwendungsmöglichkeiten der Zielsprache im südafrikanischen Kontext berücksichtigt werden, was einen Faktor darstellt, der mit der Motiviertheit der Lernenden eng zusammenhängt. 


\section{Die Rolle der von den Lernenden beherrschten Sprachen}

\subsection{Die Unterrichtssprache}

Im südafrikanischen Kontext ist der Stellenwert des Englischen von größter Bedeutung, weil Englisch als lingua franca und deswegen auch als Unterrichtssprache der Mehrheit der Südafrikaner gilt. Dabei ist es wichtig, Englischmuttersprachler von Sprechern des Englischen als L2 oder L3 zu unterscheiden, da der Grad der Automatisierung in der Unterrichts- oder Ausgangssprache für effektives Lernen entscheidend ist. In Südafrika sind nur 8,2\% (Statistics South Africa 2003:24) der Bevölkerung Englischmuttersprachler. Für alle anderen ist Englisch L2, wenn sie Glück haben, sonst eher L3, L4 oder sogar L5, je nachdem unter welchen Bedingungen sie aufwachsen. ${ }^{2}$

Die Unterrichtssprache der afrikaanssprechenden Lernenden ist in den meisten Fällen Afrikaans, was ein großer Vorteil gegenüber den anderen einheimischen Bevölkerungsgruppen ist, die in ihrer L2 oder Lx Unterricht empfangen. Diese Gruppe soll also in jeder Sprachlernuntersuchung im südafrikanischen Kontext getrennt untersucht werden. Diese Untersuchung beschränkt sich weitgehend auf die Lernenden mit bantusprachigem Hintergrund.

\subsection{Bantusprachige Lernende}

Wie automatisiert die Unterrichtssprache bei den einzelnen bantusprachigen Lernenden ist, hängt u.a. mit sozio-ökonomischen Faktoren zusammen. Diaz (1985:1381) hat in einer Untersuchung des kognitiven Vermögens von zweisprachigen Kindern festgestellt, dass eine Korrelation besteht zwischen Low English Proficiency (LEP) der Kinder und Arbeitslosigkeit und/oder niedrigem Einkommen des Familienhauptes. In Südafrika ist es, weitgehend aus historischen Gründen, vor allem in bantusprachigen Haushalten ein besonders großes Problem. Der Sprachinput im Elternhaus ist bei Kindern aus armen Verhältnissen oft auf niedrigem Niveau, was bedeutet, dass die Kinder sogar die eigene Muttersprache nur begrenzt beherrschen. Im südafrikanischen Kontext kommt die niedrige soziale Stellung der Bantusprachen, sogar in den Augen der Sprecher, als Faktor hinzu (Webb 2002). Sie gehen nicht in die Vorschule, weil sich die Eltern die Gebühren nicht leisten können und kommen ohne Englischkenntnisse in die erste Schulklasse. In dieser wichtigen Phase können sie dem Unterricht nicht folgen. Im Laufe der Zeit lernen sie mehr oder weniger Englisch sprechen und verstehen, aber so automatisiert wie bei ihren Klassenkameraden, die Englisch als Muttersprache sprechen, werden ihre Englischkenntnisse nie. Schüler mit besserem Sprachinput im Elternhaus, die in ihrer Muttersprache auf höherem Niveau Gedanken formulieren lernen, sind fähiger, in einer Zweitsprache zu lernen. In einer Schulklasse kann man also Lernende haben, die in ihrer L1 unterrichtet werden, Lernende mit gutem Sprechvermögen in der Unterrichtssprache und Lernende, bei denen ein Sprechvermögen in der Unterrichtssprache sehr begrenzt oder gar nicht vorhanden ist, je nachdem in welcher Schulphase sie sich befinden.

Auch im Unterricht des Deutschen als Fremdsprache erzielen Lernende, die in ihrer Muttersprache unterrichtet werden, oder mit besseren Zweitsprachenkenntnissen in die Schule kommen, größeren Erfolg. Diese Aussage wird durch eine Untersuchung, die an vier südafrikanischen Schulen durchgeführt wurde (Baker 2005a), bestätigt. Die afrikaanssprachigen Lernenden und die Lernenden an den Deutschen Schulen in Johannesburg und Pretoria schnitten mit Abstand besser ab als die Lernenden in einer Townshipschule in KwaZulu-Natal, die in ihrer L3/Lx unterrichtet werden und aus 
weitgehend schlechten Verhältnissen kommen. Obwohl die sozio-ökonomischen Verhältnisse der Lernenden in dieser Untersuchung nicht berücksichtigt wurden, scheint das Ergebnis der Untersuchung von Diaz (1985) auf diese Gruppen in gleichem Maße zuzutreffen.

\subsection{Sprachtypologie}

Die Psycho-Typologie der Ausgangssprache(n) und der Zielsprache spielt eine wichtige Rolle (Ringbom 1987). Als germanische Sprachen haben Deutsch, Afrikaans und Englisch vieles gemeinsam. Abgesehen von lexikalischen Ähnlichkeiten ist auch der Satzbau des Afrikaans dem Deutschen ähnlich, während im Englischen eher die Lexik eine vereinfachende Rolle einnimmt. Hufeisen (1994) hat in ihrer Monografie die lexikalischen Übereinstimmungen zwischen Deutsch und Englisch sehr ausführlich dargestellt. Aus den 15 Bereichen, die sie angibt, seien hier zwei Beispiele zitiert und um die afrikaansen Entsprechungen ergänzt.

Aus dem Bereich Wohnen:

\section{Deutsch}

Das Appartement/die Wohnung

die Toilette

das Bett

das Gas

das Glas

Umwelt:

die Natur

der Park

der Ozean

die Pflanze
Englisch

appartment

toilet

bed

gas

glass

nature

park

ocean

plant

Afrikaans
woonstel
toilet
bed
gas
glas

die natuur

park

oseaan

plant

Auch die ,falschen Freunde ' und andere Fehlerquellen werden aufgeführt und erläutert. Dazu gehört u.a. die Satzstellung. Wie aus einigen Beispielen ersichtlich wird, ist die Satzstellung des Afrikaans mit dem Deutschen fast identisch, während die englische Satzstellung einige Unterschiede aufweist.

(2) I am going to the doctor at three o' clock this afternoon.

(3) Ich gehe heute Nachmittag um drei Uhr zum Arzt.

(4) Ek gaan vanmiddag om drie uur dokter toe.

Wenn die Temporalangabe auf Position 1 gerückt wird, ergibt sich Folgendes:

(5) This afternoon I am going to the doctor at three o' clock.

(6) Heute Nachmittag gehe ich um drei Uhr zum Arzt.

(7) Vanmiddag gaan ek om drie uur dokter toe.

Auch in unterordnenden Nebensätzen ist die Satzstellung im Deutschen und im Afrikaans gleich:

(8) I am staying at home today, because I am sick.

(9) Ich bleibe heute zu Hause, weil ich krank bin.

(10) Ek bly vandag by die huis, omdat ek siek is. 
Nur in Nebensätzen mit Modalverben ist die Satzstellung im Deutschen anders als im Afrikaans, weil das Modalverb im Afrikaans an zweitletzter Stelle statt an letzter Stelle steht.

\section{Ich gehe zur Apotheke, weil ich Medikamente kaufen muss. \\ Ek gaan apteek toe omdat ek medisyne moet koop.}

Obwohl die Bantusprachen zu einer anderen Sprachfamilie gehören, lassen sich auch in diesen Sprachen Ähnlichkeiten mit dem Deutschen feststellen, die beim Erlernen des Deutschen wirksam sein könnten. Gerade die grammatischen Eigenheiten, die im Englischen und im Afrikaans fehlen, nämlich die Nomenklassifizierung und deren Einfluss auf u.a. Adjektive und Pronomen, finden sich im Zulu wieder. Baker (2005b:1-16) gibt eine detaillierte Darstellung des Kongruenzsystems und der diesbezüglichen Übereinstimmungen zwischen Deutsch und Zulu. Da es eine sehr wichtige Eigenschaft ist, die die meisten hiesigen Bantusprachen mit Deutsch gemeinsam haben, sollte man versuchen, den Lernenden diese Tatsache bewusst zu machen.

\section{Linguistisches und metalinguistisches Bewusstsein}

Dabei kommt dem metalinguistischen und dem linguistischen Bewusstsein der Lernenden und der Lehrenden eine wichtige Rolle zu. Es ist selbstverständlich, dass bei Kleinkindern und Lernenden in einem deutschsprachigen Land, dieses Bewusstsein eine geringere Rolle spielt. Wie aus der Studie von Diaz (1985) hervorgeht, ist es nicht völlig auszuschließen, weil gerade bei Kindern mit „Low English Proficiency“ mangelndes metalinguistisches Bewusstsein festgestellt wurde. Bei älteren Kindern und Erwachsenen, die in einem fremden Land eine Fremdsprache erlernen, in dem die Zielsprache von keinem, oder von sehr wenig Sprechern gesprochen wird, ist metalinguistisches Bewusstsein eine sehr wichtige Komponente. De Angelis (2007:122) beruft sich auf mehrere Studien, aus denen hervorgeht, dass metalinguistisches Bewusstsein wahrscheinlich einer der wichtigsten Faktoren ist, der einen erfolgreichen Fremdsprachenerwerb fördert. Man gibt den Lernenden mit dieser analytischen Herangehensweise ein gutes Lernmittel an die Hand, mit dem sie dann eigene, korrekte Sätze formulieren können, ohne großen Korpora ausgesetzt zu sein. Traoré (2004:38) plädiert für eine „bewusste Herangehensweise bei der Vermittlung des Deutschen als Fremdsprache“ indem er auf die Funktionsweise der menschlichen Gedächtnissysteme eingeht. Auch Sharwood Smith (1994) hat darauf aufmerksam gemacht, welche fördernde Funktion Bewusstmachung im Fremdsprachenerwerb hat.

Die rein kommunikative Lehrmethode, die sich gerade großer Beliebtheit erfreut und die in der praktischen Anwendung des südafrikanischen Schulcurriculums manchmal auf Kosten des korrekten Sprachgebrauchs geht, führt in manchen Fällen eher zu Denkfaulheit und früher Fossilierung in den etwas anspruchsvolleren Sprachlernbereichen als es eine Bereitwilligkeit zu anlytischem Denken tun würde. Es ist viel einfacher zu sagen: *Das Mädchen ist sprechen $z u$ er Mutter (The girl is speaking to her mother) als sich die richtige Verform zu merken und das richtige Pronomen mit der richtigen Endung $\mathrm{zu}$ verwenden. Weil man trotzdem verstanden wird, ist man auch nicht besonders motiviert, diese ,schwierigen Formen“ zu beherrschen. Solche Beispiele findet man ausgerechnet bei bantusprachigen Lernenden, deren Erstprache dem Deutschen in der Flektion viel ähnlicher ist als Englisch und Afrikaans. Sie benutzen die englische Krücke, wo sie eher ein Hindernis ist. Hufeisen (1994) gibt Lehrenden gute Tipps, wie Englischkenntnisse für den Erwerb des Deutschen fruchtbar gemacht werden 
können. Dies setzt aber gute Englischkenntnisse und gutes metalinguistiches Bewusstsein voraus, was im südafrikanischen Kontext nicht immer gegeben ist.

Nach Steinhauer (2006) ist Vereinfachung ein integraler Teil des Sprachlernprozesses. Das soll aber keineswegs heißen, dass es unwichtig ist, diese Phase zu überwinden und die zu erlernende Fremdsprache richtig und auf hohem Niveau sprechen zu lernen. Lehrende sollten alles daransetzen zu verhindern, dass die Lernenden in dieser Vereinfachungsphase stehen bleiben und ihre Sprache fossilieren. Die Bewusstmachung der Ähnlichkeiten von Zulu und Deutsch kann dazu beitragen, indem man den Lernenden klarmacht, dass es im Deutschen genau so wichtig ist zu wissen, zu welcher Nomenklasse ein Wort gehört, wie im Zulu. Hinzu kommt, dass das deutsche System aus nur drei Nomenklassen besteht und das Nomenklassifizierungssystem des Deutschen somit um 14 Klassen einfacher ist als das Zulu System.

Lernende aus einem bantusprachigen Kontext, die aber keine theoretisch-formalen Kenntnisse ihrer Muttersprache mitbringen, werden von Hinweisen auf die sprachlichen Übereinstimmungen zwischen Deutsch und ihrer Muttersprache nur wenig profitieren, wenn überhaupt.

Um festzustellen, welche Mittel zulusprachigen Deutschlernenden an die Hand gegeben werden können, ihre L1-Kenntnisse dem Erlernen des Deutschen nutzbar zu machen, wurde 2007 und 2008 an einer Townshipschule in KwaZulu-Natal von mir eine Untersuchung durchgeführt. Die Lernenden sind alle zulusprechende Deutschlernende der 11. Klasse, die auf Englisch unterrichtet werden. Als Auftakt zu der Untersuchung wurde die Lehrerin gebeten, die Elftklässler einen Aufsatz zum Thema „Meine Familie“ schreiben zu lassen. Aus einer Gruppe von 23 haben aber nur 9 Lernende den Aufsatz geschrieben, weil die Lehrerin nur die von ihr als die besten Deutschlernenden eingeschätzten SchülerInnen dazu aufgefordert hatte. Obwohl die Gruppe aus diesem Grund statistisch unbedeutend ist, lassen sich ganz deutliche Tendenzen feststellen. Die folgenden Beispiele zeigen, dass die SchülerInnen ihre Aufsätze auf Englisch geschrieben und danach ins Deutsche übersetzt haben.

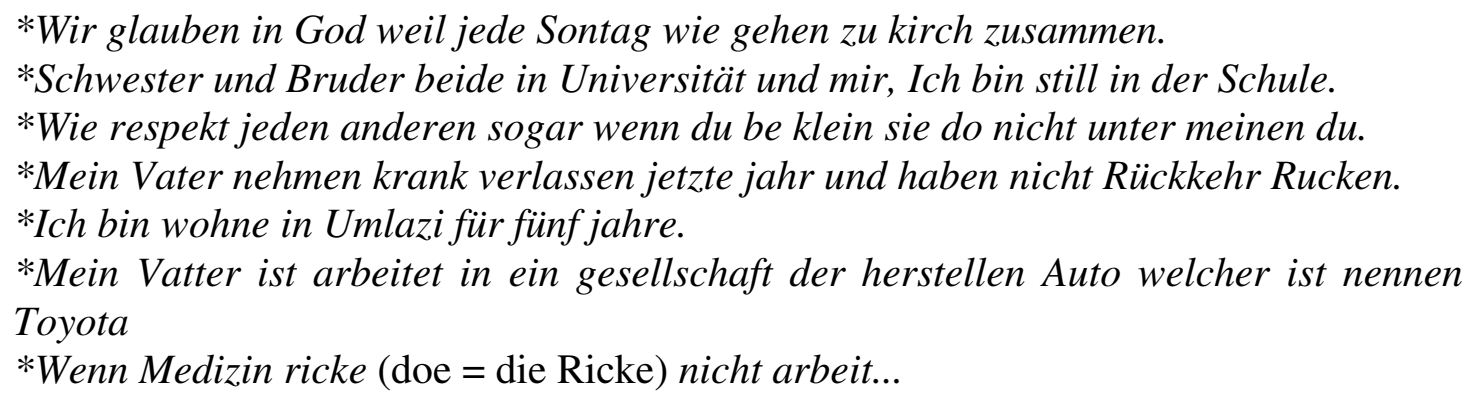

Nicht nur lexikalische, morphologische und syntaktische Einflüsse machen sich bemerkbar. Auch von den SchülerInnen als phonologisch ähnlich empfundene Elemente treten auf, wie z.B. *wie statt wir unter Einfluss des englischen we.

Nach diesem ersten Versuch folgten einige Unterrichtsstunden, in denen die Lernenden auf die Ähnlichkeiten zwischen Deutsch und Zulu aufmerksam gemacht wurden. An Hand von Beispielsätzen wurden Übereinstimmungen hervorgehoben, indem die Lernenden aufgefordert wurden, deutsche Sätze in die Muttersprache (Zulu) zu übersetzen. Danach 
bekamen sie den Auftrag, eine Bildergeschichte nachzuerzählen. Es geht um einen Dackel, der gebadet werden muss. Er rennt dem Vater und seinem Sohn davon, wird aber gefangen und muss dem Beispiel des Vaters folgen, der in die Badewanne steigt, um dem Hund zu zeigen, was er machen soll. Der Hund springt ins Aquarium statt in die Schüssel. Alle relevanten Verben und Nomen (mit Artikel) wurden an die Tafel geschrieben, bevor die Lernenden anfingen, die Geschichte zu schreiben. Trotzdem ist der Artikelgebrauch fehlerhaft, Nomen werden klein geschrieben und zeigen wird durch shau ersetzt. Es folgen einige Beispiele aus den Arbeiten von den SchülerInnen, die auch den Aufsatz über die Familie geschrieben haben:

*Die vater musst to waschen die Hund

*Die Hund Nein machen waschen en die vase (Wasser)

*Der Vater ist shau der hund in der nähe die schüssel und Vater ist springen in zu die wasser.

*Die Junge und die Hund ist waschen die vater kriege in die wasser

*Der Hund ist sitzen Morgendämmerung und der tür ist schwül

*Und der Hund sprung innenseite der Angelgerät.

*Der Vater zeigen die Hund zo krieken in die wasser außer abfall zu krieken in die wasser.

*Und die hund spalten das wasser all über die Tisch.

Weder noch vorhandene Afrikaanskenntnisse, noch die L1 der Lernenden, haben jeglichen Einfluss auf den deutschen Sprachgebrauch dieser SchülerInnen ausgeübt, obwohl sie auf Ähnlichkeiten zwischen Deutsch und Zulu aufmerksam gemacht worden waren, bevor sie den Nacherzählungsauftrag bekamen. Sie benutzten z.B. nach wie vor die „present continuous“Form des Verbs, ist arbeitet, ist nennen, ist waschen, ist sitzen, obwohl ihnen an Hand von Zulubeispielen gezeigt worden war, dass die Verbform vom Subjekt des Satzes mit bestimmt wird. Es ist nicht realistisch zu erwarten, dass die Lernenden ihre schlechten Gewohnheiten sofort durch richtigen Sprachgebrauch ersetzen könnten. Was sich jedoch deutlich bemerkbar macht, ist die Tatsache, dass ihnen metalinguistisches Bewusstsein fehlt. Sie unterscheiden bei der Benutzung des Wörterbuchs (was hier auch unnötig war, weil die Vokabeln an der Tafel standen) nicht zwischen Verben, Adverbien und Nomen. Hier sei neben Morgendämmerung für down auch * ricke für does erwähnt.

Obwohl die kommunikative Lehrmethode an den Schulen befürwortet wird, geht aus dieser Untersuchung ganz deutlich hervor, dass die Lernenden eine Übersetzungsmethode praktizieren. Längere Texte, bzw. eigene Formulierungen, werden auf Englisch konzipiert und dann ins Deutsche übersetzt. Mangelhaftes metalinguistisches Bewusstsein führt dabei zu Fehlübersetzungen, wie die zitierten Beispiele deutlich zeigen.

Eine Kooperation zwischen Sprachlehrerinnen kann auf diesem Gebiet viel leisten. Wenn die Bantusprachen an den Schulen unterrichtet würden, so dass die Lernenden die eigene Sprache wirklich kennen lernten, statt sich damit zufrieden zu geben, sie mehr oder weniger sprechen zu können, würden die Lernenden andere Sprachen um so besser beherrschen lernen. Darüber hinaus sind metalinguistische Kenntnisse ein Mittel, den Lernenden mehrere Herangehensmöglichkeiten zu bieten, die sie dann je nach Begabung einsetzen könnten. Auch hier nehmen Afrikaansmuttersprachler oft eine Sonderstellung ein, weil im Afrikaansunterricht als L1 auch metalinguistische Kenntnisse vermittelt werden. 


\section{Verwendungsmöglichkeiten}

In Südafrika gibt es kaum Gelegenheit, die deutsche Sprache zu sprechen. Auch das Internet ist nicht allen zugänglich. Diese Tatsachen wirken sich gewiss negativ auf das Sprachvermögen und die Motiviertheit der Lernenden aus. Langfristige Ziele wie Berufsmöglichkeiten bei deutschen Firmen und in der Tourismusbranche, oder sogar die hochgesteckten Ziele einiger Lernenden, in Prestigeberufen in Deutschland zu arbeiten, sind weniger wirkungsvoll als die Möglichkeit, nach der Schule mit einem deutschsprachigen Freund schwimmen zu gehen.

\section{Schlussfolgerung}

Man kann sich fragen, warum man in Südafrika noch an höheren Schulen Deutsch unterrichten will. Sollte man nicht vielmehr den Englischunterricht fördern, vor allem, da aus den Übersetzungsfehlern der Lernenden hervorgeht, dass auch die Englischkenntnisse dieser Schüler in vielen Fällen recht mangelhaft sind? Sollte man gewissen Schulen den Deutschunterricht vorenthalten? Braucht man in Südafrika Deutschkenntnisse, wenn man so weit von Deutschsprachigen entfernt lebt, und Englisch als Weltsprache anerkannt wird? Wären vielleicht Sprachenzentren, etwa nach dem deutschen Volkshochschulmodell, an denen jeder Interessent eine oder auch mehere Fremdsprachen erlernen kann, eine bessere Lösung, als das schon überfüllte Programm an den Schulen noch um DaF zu erweitern?

Klar ist auf alle Fälle, dass der Unterricht von Deutsch als Fremdsprache in Südafrika nicht ohne Probleme ist. Davon zeugen nicht zuletzt die zurückgehenden Lernerzahlen an Schulen und einigen Universitäten. Aber die Lösung ist nicht in einer Sprachverarmung und einer Abkapselung von Europa mit Hinwendung nur zu Afrika zu suchen.

\section{Anmerkungen}

1. Vgl. hierzu den Begriff der Psycho-Typologie von Ringbom (1987).

2. Webb (2000) gibt dazu sehr detailliert Auskunft. Siehe auch Chimbganda (2005) in Bezug auf die Anwendung des Begriffs ,native speaker of English“.

\section{Bibliographie}

Baker, J. 2005a. Teaching German as a foreign language with specific reference to Zuluspeaking learners. Unpublished doctoral thesis. University of Johannesburg.

Baker, A. 2005b. Noun classification and congruence in German and Zulu - a contrastive analysis. Journal for Language Teaching 39(1): 1-16.

Chimbganda, A. 2005. Profiling the "native speaker" of English: Myths and implications for ESL learning and teaching. Journal for Language Teaching 39(1):18-33.

De Angelis, G. 2007. Third or additional language acquisition. Clevedon: Multilingual Matters LTD.

Diaz, R.M. 1985. Bilingual cognitive development: Addressing three gaps in current research. Child Development 56(4): 1376-1388.

Hufeisen, B. 1994. Englisch im Unterricht Deutsch als Fremdsprache. München: Klett Edition Deutsch.

Missler, B. 1999. Fremdsprachenlernerfahrungen und Lernstrategien. Eine empirische Untersuchung. Tübingen: Stauffenburg Verlag.

Ringbom, H. 1987. The role of the first language in foreign language learning. Clevedon: Multilingual Matters. 
Sharwood Smith, M. 1994. Second language learning: Theoretical foundations. In C.N. Candlin (ed.) Applied linguistics and language study. London: Longman Group UK Limited.

Statistics South Africa. 2003. Census 2001. Census in brief. Online verfügbar unter www.statssa.gov.za; letzter Zugriff: 10.10.2009.

Steinhauer, B. 2006. Transfer im Fremdsprachenerwerb. Frankfurt a.M.: Peter Lang Verlag.

Traoré, S. 2004. Gedächtnis- und Gehirnprozesse beim Fremdsprachenlernen. Von der Wahrnehmung zur Konsolidierung fremdsprachlicher Lerninhalte. In Deutsch als Fremdsprache in Wissenschaft und Unterricht: Arbeitsfelder und Perspektiven. Festschrift für Lutz Götze zum 60. Geburtstag. Frankfurt a.M.: Peter Lang Verlag.

Webb, V. 2002. Language in South Africa. The role of language in national transformation, reconstruction and development. Amsterdam, Philadelphia: John Benjamins Publishing Company.

\section{Biographical note}

Dr Anne Baker is a Senior Lecturer in the Department of German at the University of Johannesburg. Her research focus is the teaching and learning of German as a foreign language, as well as Comparative Linguistics, specifically comparing German and Zulu. 